\title{
Saúde do idoso e o papel do odontólogo: inter-relação entre a condição sistêmica e a saúde bucal
}

\author{
Health of the elderly and the dentist's role: \\ the interrelationship between systemic conditions and oral health
}

\author{
KÉSSIA do NASCIMENTO IRINEU 1 \\ Graduanda do Curso de Odontologia da Universidade Estadual da Paraíba - UEPB \\ Jose Augusto Moura Acioli FiLho² \\ Graduando do Curso de Odontologia da Universidade Estadual da Paraíba \\ Roniery Oliveira Costa ${ }^{3}$ \\ Doutorando do Programa de Pós Graduação da UEPB e Professor da \\ Faculdade Mauricio de Nassau/Campina Grande -PB \\ Maria Helena Chaves de Vasconcelos Catão $0^{4}$ \\ Profa.Dra. em Laser em Odontologia/UFBA e Profa. Dra. do \\ Programa de Pós Graduação da UEPB
}

\begin{abstract}
RESUMO
Introdução: $O$ envelhecimento populacional é uma realidade observada em diversos países mundo e este amadurecimento traz consigo uma série de implicações que afetam as sociedades em suas diversas esferas organizacionais, interferindo de forma direta na organização do país. Boa parte dos profissionais de saúde mostra-se despreparada para prestar assistência individualizada e humanizada, fato justificado pela ausência de componentes curriculares, de cunho odontogeriátrico, na formação profissional. Este período é marcado pela prevalência de doenças crônico-degenerativas e estas desencadeiam debilidades, deficiências e muitas vezes necessidade de cuidados especiais por parte de cuidadores e familiares. Entretanto, deve-se lembrar que existe uma relação direta entre saúde bucal e saúde geral do indivíduo e vice-versa. As doenças, assim como as condições que levem ao acamamento, devem ser trabalhadas de forma conjunta para que se alcance o bem-estar dos idosos. Proposição: O objetivo desta revisão de literatura é refletir sobre a inter-relação entre a condição sistêmica e a saúde bucal e apontar o papel do odontólogo na saúde do idoso. Materiais e métodos: A bibliografia foi obtida no banco de dados da Lilacs, Medline e Scielo utilizando os seguintes descritores: saúde do idoso; assistência à saúde; odontologia geriátrica; assistência odontológica para idosos; saúde bucal, indexados de 2008 a 2014. Conclusão: É imprescindível que o profissional esteja comprometido, buscando aperfeiçoamento das habilidades e atualização dos conhecimentos para assim prestar um atendimento de qualidade e que vá ao encontro das necessidades dos idosos.
\end{abstract}

Palavras-chave: saúde do idoso; assistência à saúde; odontologia geriátrica; assistência odontológica para idosos; saúde bucal.

\begin{abstract}
Introduction: Aging population is a reality observed in many countries around the world and this maturity brings along a number of implications that affect societies in their various organizational levels, interfering directly in the country's organization. Many health professionals are unprepared to provide individualized and humanized care, which may be explained by the absence of geriatric dentistry in their professional training. This period is marked by the prevalence of chronic degenerative diseases that trigger weaknesses, deficiencies, and often need that special care be offered by caregivers and family members. However it should be remembered that there is a direct relationship between the individual's oral health and general health and vice versa. Diseases and conditions that lead the elderly to bed confinement must be worked jointly to achieving the their well-being. Purpose: The purpose of this review is to reflect on the interrelationship
\end{abstract}


between systemic conditions and oral health and to point out the dentist's role in maintaining the elderly's health. Materials and methods: Literature on this subject was obtained in Lilacs, Medline and Scielo databases using the following keywords: Elderly's Health; Health care; Geriatric dentistry; Dental care for the elderly; Oral Health, indexed from 2008 to 2014. Conclusion: It is essential that the professional is committed to seek the improvement of skills and update his/her knowledge so as to provide a quality service that meets the elderly's needs.

Keywords: Elderly's Health; health care; geriatric dentistry; dental care for the elderly; oral health.

\section{INTRODUÇÃO}

O envelhecimento populacional é uma realidade observada em diversos países ao redor do mundo. Ele traz consigo uma série de implicações que afetam as sociedades em suas diversas esferas organizacionais (políticas, econômicas, culturais e sociais) interferindo, portanto, de forma direta na organização do País, ${ }^{1}$ assim como no comportamento profissional dos trabalhadores, principalmente daqueles ligados à área da saúde..$^{2,3}$

No Brasil, esse novo perfil demográfico está associado a dois fatores, sendo estes a queda dos níveis de fecundidade e o aumento da expectativa de vida da população. Como consequências desse processo ocorreram mudanças nos padrões de morbidade, invalidez, bem como de mortalidade. ${ }^{4,5}$

A presença de doenças crônicodegenerativas é comum ao envelhecimento e estão intimamente ligadas a esse processo. Dentre as principais enfermidades crônicas enfrentadas pelos idosos encontram-se as doenças cardiovasculares, diabetes, aterosclerose, doença pulmonar obstrutiva crônica (DPOC) e osteoporose, sendo a hipertensão e o diabetes as de maior frequência nessa faixa etária. ${ }^{6}$

Mal de Alzheimer, Parkinson e outras condições que levem ao acamamento podem interferir na autonomia do idoso, tornando-o dependente de outros indivíduos. Portanto, o atendimento por parte de cirurgiões-dentistas deve ser diferenciado, bem como a atenção especial da família e/ou cuidadores. Forma-se, portanto, uma tríade de cuidados para com o idoso dependente. Esta tríade tem por objetivo preservar a condição de saúde do indivíduo, mantendo-a o mais saudável possível. 2,5,6
Essa fase também promove maiores dificuldades em relação à acessibilidade, visto que os transportes públicos, ambientes $\mathrm{e}$ serviços mostram-se inadequados às condições físicas dos idosos, que muitas vezes encontramse debilitados, necessitando de medidas que favoreçam maior acessibilidade ao serviço., ${ }^{1,2}$

Dentre as principais dificuldades encontradas pelos idosos dependentes para a utilização do serviço odontológico no consultório podemos citar: transportes públicos, calçadas e ambientes inadequados, consultório mal adaptado, longo tempo de espera para atendimento, ausência de informações sobre os serviços odontológicos disponíveis, ausência de equipamentos e dificuldades na mobilidade pela diminuição dos sentidos. ${ }^{1}$

O profissional deve estar atento às particularidades inerentes ao idoso e às suas necessidades, tanto em relaçãoàacessibilidade ao serviço, como também às principais condições orais que estes apresentam, para poder prestar um atendimento de qualidade. ${ }^{7}$

Dentre as alterações orais mais encontradas nos idosos, temos: redução do fluxo salivar, xerostomia, estomatites protéticas, língua fissurada, língua saburrosa, candidose, queilite angular, hiperplasia fibrosa, ${ }^{6}$ cárie, doença periodontal, edentulismo etc., ${ }^{3}$ podendo ser potencializadas por determinadas condições sistêmicas bem como exercer influências sobre elas. ${ }^{2}$

Portanto, o presente estudo teve por objetivo refletir sobre a condição sistêmica e a saúde bucal e sua influência na qualidade da saúde do idoso, além de discutir sobre a importância de um cirurgião-dentista bem capacitado na equipe interdisciplinar de atenção a este paciente. 


\section{Material e mÉtodos}

Este estudo baseou-se em uma revisão de literatura nos bancos de dados da Lilacs, Medline e Scielo, utilizando os critérios de inclusão à língua portuguesa e inglesa, indexação nas plataformas entre anos de 2008 a 2014 e disponíveis em forma completa. Foram utilizados para a pesquisa os descritores disponíveis no DeCS: Saúde do Idoso; Assistência à saúde; Odontologia geriátrica; Assistência odontológica para idosos e Saúde Bucal.

Como critério de exclusão utilizou-se qualquer outra língua que não a portuguesa e a inglesa e artigos que não fossem relacionados ao tema, além daqueles disponíveis apenas em forma de resumo.

O presente artigo foi solicitado como requisito para a conclusão do componente curricular Odontologia Geriátrica, disponível na grade curricular do curso de Odontologia da Universidade Estadual da Paraíba (UEPB), não sendo necessário, para este fim, ser realizado em forma de revisão sistemática.

\section{REVISÃo dA LITERATURA}

O conceito de envelhecimento tem se modificado com os anos, passando de um amadurecimento caracterizado pela inatividade profissional, abandono social, morbidades indesviáveis, edentulismo certo (perda inevitável de dentes, seja parcial ou total) e mortalidade em tenra idade precoce, a um novo conceito de envelhecimento saudável e manutenção ativa destes indivíduos na sociedade. ${ }^{1,3,8}$

A autopercepção dos indivíduos sobre sua qualidade de vida é essencialmente importante, visto que é por meio desse parâmetro que ele enxerga sua condição de saúde e compreende sua necessidade de serviços de saúde ${ }^{9}$ necessidades nutricionais, ${ }^{10}$ físicas, psicológicas e sociais, dentre outras. ${ }^{11}$

A mudança no perfil demográfico brasileiro vem ocorrendo de forma acelerada. De acordo com projeções estatísticas, em 2025 a população idosa corresponderá a $15 \%$ da população brasileira, ou seja, 32 milhões de habitantes. Levando-se em consideração estes dados, percebe-se a necessidade de oferecer um cuidado holístico a estes indivíduos, ${ }^{11}$ visto que a cada dia eles vêm construindo um perfil diferenciado de autopercepção de sua condição oral. ${ }^{10}$

As doenças bucais, assim como a ausência de elementos dentários, representam um impacto na vida cotidiana do indivíduo, interferindo em funções - como a estética - e também em suas relações sociais. No entanto, este impacto é sentido de forma distinta pelos indivíduos a partir de sua percepção sobre sua condição oral e envelhecimento. ${ }^{11,12,13}$

$\mathrm{Na}$ atualidade ainda há uma parcela expressiva de indivíduos edêntulos que não utilizam próteses dentárias, ou as utilizam, porém mal adaptadas ou possuindo uma condição de saúde oral deficiente. ${ }^{13}$ Tal fato ocorre principalmente com indivíduos considerados em situação de risco, ou seja, inseridos em uma cultura que negligencie o cuidado com a saúde bucal, e que possuam baixa condição econômica e educacional. ${ }^{3}$

Outro aspecto importante a ser mencionado é que muito embora a prática da higiene oral, por meio da escovação mecânica, seja tão disseminada em nossa cultura, esta apresenta números menos expressivos quando se associa o número de idosos com as demais faixas etárias da sociedade. ${ }^{14} \mathrm{Tal}$ dado aponta para a necessidade de ações que promovam o autocuidado e a procura por serviços de saúde, com a intenção de manter os elementos na cavidade oral e em bom estado. ${ }^{15}$

Políticas públicas destinadas à população idosa são imprescindíveis, principalmente de âmbito odontológico. Estas ações devem ser realizadas de forma mais ampla e com foco na educação e promoção da saúde, a fim de beneficiar a população do País como um todo.

Outro aspecto importante a ser ressaltado é que a condição de saúde oral do idoso acamado tem repercussões na vida dos membros da família, e estes devem estar dispostos a assistir o idoso em suas necessidades de saúde e 
favorecer a recuperação de sua qualidade de vida, mesmo que esta dedicação reduza o tempo destes familiares na execução de seus próprios afazeres individuais. ${ }^{16}$

\section{Dıscussão}

O aumento da faixa etária acima dos 60 anos $^{11}$ tem favorecido o crescimento do mercado odontológico, assim também como o aumento da busca por profissionais com uma formação mais completa, voltada à atenção odontogeriátrica. ${ }^{2,4,6}$

Estes profissionais têm um olhar diferenciado no atendimento a esse público, em virtude de desenvolverem suas atividades correlacionando aspectos da condição sistêmica e saúde oral e por compreenderem que a saúde bucal tem influência na saúde geral, assim como o inverso. ${ }^{2,3,6}$ Estes profissionais ainda buscam prestar uma assistência de forma multiprofissional, multidisciplinar e transdisciplinar, a fim de promover um tratamento mais efetivo para recuperação ou manutenção da condição de saúde do indivíduo. ${ }^{3,6}$

Os idosos apresentam uma grande prevalência de doenças crônicas e, consequentemente, fazem uso da polifarmácia. Estes fatores, somados a uma exposição aos fatores etiológicos das doenças orais, favorecem a instalação e/ou desenvolvimento destas, ${ }^{2,7}$ trazendo um impacto à saúde bucal e ao tratamento odontológico. ${ }^{3}$ Portanto, justifica-se ainda mais a necessidade de profissionais preparados para tratar esse público e de instituições que se comprometam com a formação profissional ligada também às necessidades específicas dos idosos..$^{2,4,6}$

Sabe-se, portanto, que a maioria das universidades brasileiras não oferece o componente curricular Odontogeriatria (Odontologia geriátrica). Quando está presente na grade curricular, na maior parte dos casos apresentase como um componente optativo, não alcançando todos os discentes do curso. Assim, fica uma lacuna na formação profissional do acadêmico, que, uma vez formado, não terá a fundamentação teórica de que precisará para oferecer um tratamento diferenciado que atenda às necessidades específicas desse grupo etário que só vem crescendo com o passar dos anos.,

Os idosos dependentes, semelhantemente aos não dependentes, necessitam de tratamento odontológico. Como alternativa ao tratamento em consultório, encontra-se o atendimento odontológico em domicílio (home care) para os idosos dependentes. Este tratamento, por sua vez, para ter o resultado esperado, exige uma corresponsabilidade entre os familiares (responsáveis pelo idoso), cuidadores, demais profissionais da equipe multiprofissional e o cirurgião-dentista. ${ }^{2,3,5,6}$

Ao odontólogo compete orientar, supervisionar, treinar familiares e cuidadores (assim como o idoso, quando cooperativo) sobre os cuidados de higiene oral, motivação e realização de procedimentos menos complexos (urgências odontológicas exames de rotina, tratamento restaurador atraumático - ART, tratamento periodontal, confecção, reparos e reembasamento de próteses já existentes etc.), visto que pela debilidade dos pacientes certos procedimentos são raros. O profissional deve melhorar e/ou inserir uma rotina de higiene oral e manter um relacionamento assertivo e sincero com o idoso a família e a equipe multiprofissional. ${ }^{5,6}$

Quanto ao cuidador, este tem um papel fundamental na realização da higiene oral do paciente. Sua adesão à rotina de higiene oral é fundamental para manter a saúde bucal do paciente. Em relação ao paciente, este pode contribuir ou não para a sua higiene; no entanto seu estado cognitivo é fundamental, visto que ele deve apresentar ao cirurgiãodentista suas expectativas e receios para que o profissional possa trabalhar em cima destes dois aspectos. . $^{2,5} 6$

$\mathrm{Na}$ Estratégia de Saúde da Família (ESF), os profissionais devem realizar visita domiciliar aos idosos dependentes, atendendo as populações menos favorecidas e que não tenham condição financeira para cobrir os gastos com home care e/ou frequentar 
um consultório particular. No entanto, os programas de saúde bucal em nível coletivo ainda são tímidos, ${ }^{3}$ não comportando o atendimento a essa parcela da população de forma adequada. Estas pessoas, quando precisam de atendimento odontológico, fazemno em âmbito particular, o que muitas vezes não é possível em razão de dificuldades de mobilidade e, principalmente, financeiras. . $^{3,5,6}$

A postura profissional deve buscar compreender melhor o contexto de vida destes idosos dependentes e buscar uma relação mais positiva e empática ante este grupo, buscando proceder, de acordo com a realidade da casa e do serviço, segundo as necessidades específicas dos idosos. .,3,4,6 $^{2}$

Muito embora as políticas públicas, nas esferas federal e municipal, voltadas às necessidades de saúde bucal tenham permitido melhoras na condição oral destes indivíduos, uma grande parcela da população ainda necessita de atendimento odontogeriátrico de forma direta, tanto de cunho hospitalar como das unidades básicas da ESF e nas universidades de Odontologia de todo o País para que assim se possa mudar o atual prisma das condições bucais do idoso..$^{3,6}$

Com o passar dos anos, a autopercepção da condição de saúde bucal vem sendo alterada por meio de ações educativas e preventivas realizadas pelos serviços de saúde públicos, privados e pelas universidades de Odontologia. 3,6,17 Essa consciência dá-se, por sua vez, por meio dessas políticas relacionadas ao envelhecimento saudável. No entanto, ainda há uma parcela da população que está passando pela produção social do processo saúde-doença, ou seja, o meio em que vive favorece o desenvolvimento de determinadas condições bucais e gerais. ${ }^{17}$

$\mathrm{Na}$ literatura, o processo saúde/ doença tem sido ligado a fatores micro e macrodimencionais, ou seja, associados a fatores sociais, demográficos, econômicos, psicossociais e comportamentais além da autopercepção dos indivíduos sobre sua condição de saúde bucal. ${ }^{3,17}$
A procura por atendimentos odontológicos vem aumentando nessa faixa etária, que busca manter, melhorar ou recuperar a condição de saúde bucal e, consequentemente, sentir-se socialmente aceito. ${ }^{3}$ No entanto as estratégias de educação em saúde não devem ser abandonadas, visto que ainda há um número expressivo de indivíduos que necessitam ser educados quanto à necessidade da assistência odontológica. 2,4,17

Em decorrência da mudança do perfil demográfico da população mundial, um novo mercado de trabalho vem ganhando espaço - a odontologia geriátrica. Portanto cada dia mais se percebe a necessidade de profissionais prontamente capacitados a atender a essa demanda populacional, com características e condições particulares em relação aos demais perfis etários da população. Em decorrência dessa necessidade, o serviço odontológico clama por profissionais capacitados para trabalharem de forma efetiva no atendimento a estes pacientes e por instituições que despertem este interesse nos discentes. . $^{2,3,4,5}$

\section{Conclusões}

Os estudos apontam que as populações estão envelhecendo e esse processo implica mudanças na organização administrativas dos países assim como na oferta de bens e serviços prestados as populações. Estas mudanças têm por objetivo proporcionar um envelhecimento saudável por meio de políticas públicas de promoção, prevenção, educação e recuperação da saúde.

A odontologia geriátrica tem ganhado mercado de trabalho, visto que a procura dos serviços odontológicos tem aumentado, por parte dos idosos e de seus familiares. Portanto justifica-se a necessidade de profissionais que ofereçam um atendimento de qualidade e que, quando necessário, atuem de forma multiprofissional e multidisciplinar, visto que é notável a inter-relação entre a condição sistêmica e a oral. Estes profissionais também devem constantemente se atualizar para que 
este atendimento venha a ser mais e mais efetivo na busca pela obtenção da melhor condição de saúde do indivíduo.

\section{REFERÊNCIAS}

1. Brasil. Ministério da Saúde. Estatuto do idoso. $3^{a}$ edição. Brasília: Ministério da Saúde; 2012.

2. Saintrain MVL; Vieira LJES. Saúde bucal do idoso: abordagem interdisciplinar. Ciência \& Saúde Coletiva. 2008; 13(4):1127-32.

3. Filho HRK, Koch LFA, Bisinelli JC, Kusma SZ, Alanis LRA, Moysés ST. Uma década da odontogeriatria brasileira. Arch Oral Res. 2011 Sept.-Dec.; 7(3):295-310.

4. Melo ALSF, Erdmann AL, Caetano JC. Saúde bucal do idoso: por uma política inclusiva. Texto Contexto Enferm. out.-dez. 2008; 17(4): 696704.

5. Souza ICP, Silva AG, Quirino ACS, Neves MS, Moreira RL. Perfil de pacientes dependentes hospitalizados e cuidadores familiares: conhecimento e preparo para as práticas de cuidado domiciliar. Rev Mim Enferm. jan.-mar. 2014; 18(1): 164-72.

6. Rocha DA, Miranda AF. Atendimento odontológico domiciliar aos idosos: uma necessidade na prática multidisciplinar em saúde. Revisão de Literatura. Rev Bras Geriatr Gerontol. nov. 2013; 16(1): 181-9.

7. Lucena AAG, Costa EB, Alves PM, Figueredo RLQ, Pereira JV, Cavalcanti AL. Fluxo salivar em pacientes idosos. Rev Gaucha Odontol. jul.-set. 2010; 58(3): 301-5.

8. Martins AMEBL, HaikalDAS, Neto PES, Oliveira MP, Eleutério NB, Ferreira RC. Benefícios da proposta da Organização Mundial da Saúde para avaliação da necessidade de tratamento dentário entre idosos. Arq Odontol. out.-dez. 2012; 48(4): 263-8.

9. Vasconcellos LCA, Junior RRP, Teles JBM, Mendes RF. Autopercepção da saúde bucal de idosos de um município de médio porte do nordeste brasileiro. Cad. Saúde Publ. jan.-fev. 2012; 28(6): 1101-10.

10. Ribeiro AFL, Leal MC, Marques APO. Importance of geriatric dentistry to elderly nutrition. Rev
Gaucha Odontol. abr.-jun. 2012; 60(2): 241-6.

11. Domingos PAS, Moratelli RC, Oliveira ALBM. Atenção odontológica integral ao idoso: uma abordagem holística. Rev Odontol. Univ. Cidade de São Paulo. mai.-ago. 2011; 23(2): 143-53.

12. Issrani $R$, Ammanagi $R$, Keluskar V. Geriatric dentistry: meet the need Rev Gerodontology. mar. 2012; 29: e1-e5.

13. Leitão RFA, Azevedo AC, Bonan RF, Bonan PRF, Forte FDS, Batista AUD. Fatores socioeconômicos associados à necessidade de prótese, condições patológicas e autopercepção da saúde bucal em população idosa institucionalizada. Pesq Bras Odontoped Clin Integr. abr.-jun. 2012; 12(2):179-85.

14. Uslar ISDV, Curvino MPF, Groisman S, Senna MAA. Percepção sobre saúde e saúde bucal em uma população de idosos no município de Araruama/RJ. Rev Bras Odontol. jan.-jun. 2011; 68(1): 101-6.

15. BelotiAM, Schwab BL, Bertipaglia T, Nishimori LE, Molena-Fernandes CA. Avaliação das condições de saúde bucal de idosos institucionalizados em asilos públicos de Maringá-PR. Cienc Cuid Saude. jan.-mar. 2011; 10(1) 96-100.

16. Hiller M, Bellato R, Araújo LFS. Cuidado familiar à idosa em condição crônica por sofrimento psíquico. Esc Anna Nery. jul.-set. 2011; 15(3): 542-9.

17. Gabardo CLG, Moysés ST, Moysés SJ. Autopercepção da saúde bucal conforme o perfil de impacto da saúde bucal (OHIP) e fatores associados: revisão sistemática. Rev Panam Salud Publica. ago.-dez. 2013; 33(6): 439-45.

\section{1,2,3: Elaboração do artigo}

4: Orientação do trabalho

Submetido em: 11/06/2015

Aceito em:05/10/2015 